\title{
FORJANDO LA ESPIRITUALIDAD CRISTIANA
}

El disCURSo EVANGELIZAdor EN LA Lima DEL SIGLO XVII

\begin{tabular}{c}
\hline Maria Elizabeth Puertas Porras \\
Universidad Católica Sedes Sapientiae \\
mpuertas@ucss.edu.pe
\end{tabular}

Fecha de recepción: agosto de 2019 Fecha de aceptación: diciembre de 2019

ReSUMEN: El presente artículo busca describir las características del discurso evangelizador con respecto a la explicación de la Fe y la naturaleza de Dios en el Sermonario del extirpador de idolatrías Fernando de Avendaño. Los sermones publicados en 1649 están orientados, desde nuestra perspectiva, a consolidar la conciencia espiritual cristiana entre la población originaria de la Lima virreinal que aún mantenía creencias religiosas tradicionales. Buscamos evidenciar cómo una prédica que apela a la razón, desde la observación de la realidad, lleva a los oyentes a reflexionar y eventualmente interiorizar conceptos fundamentales del cristianismo.

Palabras Clave: Evangelización, sermones, historia de América, teología cristiana, espiritualidad, prédica, idolatría

María Elizabeth Puertas Porras es historiadora egresada de la Universidad Nacional Mayor de San Marcos. Ha colaborado con artículos en publicaciones del Congreso de la República, la revista Investigaciones Sociales de la UNMSM, la Revista Studium Veritatis de la Universidad Católica Sedes Sapientiae, entre otras publicaciones de esta universidad. A la fecha investiga acerca de la teología cristiana del siglo XVII y su influencia en la transformación de los cultos tradicionales andinos. 


\title{
FORGING CHRISTIAN SPIRITUALITY. THE EVANGELIZING DISCOURSE IN LIMA IN THE 17TH CENTURY
}

\begin{abstract}
Aвstract: The current paper seeks to describe the characteristics of the Evangelistic discourse regarding the explanation of the Faith and the nature of God in the Sermonary of the idolatry excerptor Fernando de Avendaño. The sermons, published in 1649, are oriented from our perspective to consolidate the Christian spiritual conscience among the original population of the viceregal Lima that still held tradicional beliefs. We seek to show how a preaching that invokes to reason from the observation of reality leads listeners to reflect and eventually internalize fundamental concepts of Christianity.
\end{abstract}

Kerwords: Evangelism, sermons, American history, Christian theology, spirituality, preach, idolatry.

\section{Introducción}

a cristianización de las poblaciones conquistadas durante el siglo
XVI fue un proceso caracterizado por un avance lleno de vicisitudes,
marchas, contramarchas y dificultades procedentes de diverso origen. Sin embargo, lo que resulta innegable, como lo afirmó en su momento el antropólogo jesuita Manuel Marzal, ${ }^{1}$ es que se produjo una transformación religiosa, decididamente marcada por la adopción del cristianismo. Ya desde

1 El antropólogo jesuita Manuel Marzal dedicó buena parte de su vida académica al estudio de las religiones andinas y a sustentar la tesis de la transformación religiosa del mundo andino. 
las primeras décadas de la conquista, aun en medio de conflictos, epidemias y un caos generalizado, el mensaje cristiano se fue expandiendo a tal punto que inclusive movimientos nativistas, contestatarios del nuevo régimen, identifican a sus líderes con nombres y atributos cristianos (Pease, 1991 p. 340). Sostenemos que si bien la adopción del cristianismo en esta primera etapa distó mucho de darse en términos idóneos desde la perspectiva doctrinal, sí tuvo un impacto suficientemente fuerte como para dar inicio a un proceso de transformación que ya no tenía marcha atrás. En el lapso de cien años a partir de la llegada de los españoles, prácticamente no existía población en el territorio del virreinato del Perú donde no se conociera la fe cristiana.

La adopción del cristianismo fue integrándose a muchas prácticas tradicionales que, dado el carácter politeísta de la población andina, no eran directamente percibidas como contrarias a la nueva religión. Múltiples factores contribuyeron a que este actuar se normalizara dando lugar a una nueva identidad religiosa que tomaba elementos de ambas fuentes tal como había ocurrido durante la cristianización de Europa y otros territorios. A inicios del siglo XVII esta situación parecía estar establecida hasta que tensiones existentes entre los sectores involucrados en la evangelización indígena2 dieron lugar a que se evidenciase el tema de la permanencia de los cultos prehispánicos como un instrumento para afianzar el control sobre estos sectores con un impacto importante sobre la población originaria. Así el siglo XVII trajo consigo el gran desafío de plantear la necesidad de

2 Se trata de un momento en el cual existen varios niveles de tensión. El primero de ellos entre la corona española y el clero regular por las imposiciones del Real Patronato; el segundo, entre el clero y los hacendados que se disputan a la población por la creación de nuevas doctrinas y el que se da entre los doctrineros y la población a su cargo. Véase Pease (1991, pp. 338 -339). 
una nueva evangelización, dándose inicio a las Campañas de extirpación de idolatrías cuyo objetivo sería eliminar todo vestigio de estas creencias.

Entre las estrategias utilizadas para desarrollar tan ambiciosa tarea se recurrirá a la prédica de modo preferente, pues se sostiene que solo a través de ella podrían esclarecerse las dudas que habían propiciado la permanencia de muchos errores en el ejercicio de la Fe.

Entre los autores de este tipo de textos, optamos por elegir al canónigo Fernando de Avendaño y su obra Sermones de los misterios de nuestra Santa fe católica en lengua castellana y la general del inca (1649)3, debido no solo a la amplia experiencia de su autor en el tema de la idolatría sino, particularmente, por tratarse de un texto que contó con la aprobación y el apoyo decidido para su publicación por parte de las autoridades eclesiásticas en un momento clave, previo al inicio de una nueva serie de campañas de extirpación de idolatría en la zona de Cajatambo al norte de Lima. Considerando que este territorio ya había sido objeto de estas expediciones a inicios del siglo XVII, y que el autor de los sermones había participado de ellas, resulta de particular interés analizar el discurso desarrollado para una población que conocía y, o no había asimilado la prédica de la primera expedición o había recaído nuevamente, existiendo en ambos casos, entendemos, un interés en redactar textos que fueran accesibles y cumplieran su cometido: llevar a cabo una auténtica conversión.

Aquí nos ocuparemos de analizar los primeros sermones de la obra que abordan el tema de la Fe y la naturaleza de Dios, contenidos de difícil aprehensión para los naturales pero que, gracias al conocimiento adquirido por su autor durante su larga experiencia de contacto con la población

3 Las referencias a los textos originales se realizarán según lo establecido en las Normas de transcripción paleográfica. 
andina, encuentran en estos textos los medios para llegar a la conciencia de los oyentes.

Nacido en Lima en 1576, Fernando de Avendaño fue un clérigo de sólida formación intelectual. Educado en el colegio jesuita de San Martín, recibe una preparación esmerada. Tuvo entre sus maestros al jesuita José de Arriaga, experto en el tema de la idolatría y autor del manual para clérigos de doctrina La extirpación de la idolatría en el Perú (1621). Avendaño estudia en la Universidad de San Marcos llegando a convertirse en un reconocido académico. Aquí ocupa en 1605 las cátedras de Artes. y Teología y, años después, el cargo de rector hasta en dos oportunidades (Guibovich 1993 p. 169). En 1606 asume la doctrina de San Pedro de Casta y en 1612 es designado juez visitador de idolatrías por el arzobispo Lobo Guerrero, dando con ello inicio a lo que será su larga carrera al frente de estas campañas. En 1620 se desempeña como cura de Santa Ana y la parroquia del Sagrario. Por designación del arzobispo Gonzalo del Campo asume, entre 1638 y 1641, el cargo de provisor en el Arzobispado. Este año arriba a Lima el arzobispo Pedro de Villagómez, con quien Avendaño establece una cercana relación que se verá expresada en la publicación de sus sermones en edición bilingüe (castellano - quechua) en 1649. La exitosa carrera de Avendaño culmina con su nombramiento como arzobispo para la diócesis de La imperial en Chile, puesto que no llega a ocupar, pues fallece en 1655 .

Como vemos Fernando de Avendaño contó con todas las condiciones para desarrollar un discurso orientado a afianzar entre la población indígena la formación de una conciencia cristiana.

El sermonario de Avendaño está estructurado en conformidad a lo dispuesto por el Tercer Concilio Limense (Acosta, 2014, p. 376). El texto consta de dos partes. En la primera, conformada por diez sermones, 
se busca definir al Dios cristiano, explicar la naturaleza de la Fe y establecer claras diferencias entre el Dios cristiano y las deidades andinas. Además se explica el origen de la humanidad y cómo esta cayó en el pecado haciéndose necesaria la venida de Cristo. En la segunda parte, conformada por veintidós sermones, se explican los sacramentos en relación a los mandamientos de la Fe, la necesidad de la oración, la muerte y el juicio final.

En esta obra, Avendaño hace gala de su amplia experiencia en lo referente al conocimiento de las modalidades del culto ancestral, pues se refiere a ellas en detalle empleando términos familiares al común de sus oyentes con el fin de evidenciar la debilidad de estas creencias y el peligro que constituían para el alma de los cristianos.

A diferencia del caso del texto publicado por el reconocido extirpador de idolatrías Francisco de Ávila, los sermones de Avendaño contaron con el respaldo total de las autoridades eclesiásticas para su publicación4. Esto debe haber reflejado el interés de la Iglesia tanto en insistir en los puntos expuestos por Avendaño como en demostrar la aplicación que se hacía de las disposiciones del Tercer Concilio (Lisi, 2008).

Aunque posiblemente estos sermones ya tenían varias décadas de predicación y el contexto había cambiado (Acosta, 2014, p. 378), el tema de fondo seguía siendo el mismo: formar la conciencia cristiana de la población originaria. Un proceso, que sin duda, habría de tener sus etapas y que, en estos momentos, contaba con mejores condiciones para concretarse en la medida que el mensaje estaba dirigido a una nueva generación de oyentes nacidos durante la primera evangelización y formados en contacto con la Fe.

Proponemos que a través de la prédica se busca consolidar una conciencia cristiana a partir de la reflexión en una población que ya se encuentra preparada para ello, pues ha pasado la etapa de adquirir y poner en 4 Véase Duviols (2003, p. 96). 
práctica el cristianismo en sus aspectos formales. De lo que se trata ahora es de ir más allá en la búsqueda de un convencimiento que lleve a los naturales a cuestionar las creencias de sus ancestros y, en definitiva, abandonarlas a partir de la comprensión de conceptos esenciales del cristianismo como los que abordaremos más adelante.

\section{El Sermón como Método de Evangelización}

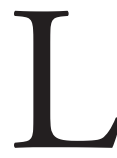

os sermones, tradicional sistema de evangelización desde el medioevo, constituirán la base de la prédica religiosa en esta etapa de la cristianización pues están orientados a convencer a los fieles a través de argumentos razonados a partir de ejemplos de su propia experiencia (Cerdán, 1996, p. 26). Los sermones buscan llevar a la reflexión y al esclarecimiento de las dudas que aún persistían y no habían logrado dilucidarse a lo largo del tiempo ya transcurrido. Las causas podemos hallarlas en diversas circunstancias que derivaron en que se privilegiaran las demostraciones externas de cristiandad frente a una auténtica conversión, objetivo central de la evangelización 5 .

La estructura de los sermones se inicia con la presentación de un tema, seguido de una explicación bajo la forma de preguntas, (que el predicador asume debían hacerse los oyentes), y respuestas dirigidas a develar las contradicciones entre el cristianismo y otros cultos6.

5 Esta situación es comentada por el clérigo José de Acosta cuando se refiere al poco conocimiento de Cristo por parte de los indios convertidos. Véase Acosta (1954).

6 Una de las estrategias empleadas durante la primera etapa de la evangelización se caracterizó por vincular lo sagrado ancestral con el cristianismo, surgiendo asociaciones que a la larga contribuyeron a mantener en la mente de los catequizados la imagen de las deidades andinas. 
El clérigo jesuita José de Arriaga destaca la necesidad de la predicación como medio preferente para combatir la idolatría, pues si bien se refiere a la permanencia de las huacas e ídolos como causas de esta, destaca especialmente el problema de "la falta de dotrina, y predicación; la qual se a de endereçar, no sólo en plantar en sus coraçones las verdades de la Religión Christiana, sino a arrancar de ellos las maleças, y raízes de sus errores" (Arriaga, 1621, p. 104).

Asimismo, insistía en la necesidad que los textos estuvieran redactados en un lenguaje acorde a la comprensión de los fieles, diciendo "Los sermones an de ser proporcionados a su capacidad, arguyéndoles, y convenciéndoles con más razones naturales, que ellos entiendan, que con pasos delicados de la escritura" (Arriaga, 1610, p. 127).

Siguiendo el modelo de la metrópoli, también aquí, el sermón se convierte el medio preferente para "convertir las almas y reformar las costumbres" (Cerdán, 1996, p. 25).

\section{El Discurso en torno a la Necesidad de la Fe}

\subsection{La Fe como don de Dios}

El primer sermón de Avendaño corresponde a la reflexión en torno a la necesidad de la Fe. Como veremos, busca que los oyentes reconozcan en sí mismos la presencia de Dios y se identifiquen como sus criaturas, pues el Creador les ha otorgado un don que les permite reconocerse como tales. Así se explica "La Fe no es otra cosa sino un conocimiento sobrenatural de 
Dios. Es una lumbre que Dios nos infunde y pone en el entendimiento del hombre para conocer a Dios" (Avendaño, 1649, f. 2).

La intención aquí es que los oyentes identifiquen este don a través de la razón. Para ello, se hará uso de ejemplos cotidianos comparando la Fe con el guía que evita que un viajero se extravíe (Avendaño, 1649, f. 2), resaltando que así como al ser humano Dios le ha otorgado los recursos necesarios para satisfacer sus necesidades corporales, más aún le habría de otorgar lo necesario para el bien de su alma que le permitirá conocerlo y encontrarse con Él, aspiración suprema del hombre (Avendaño, 1649, f.2).

Otro medio del que se dispondrá para comprender la existencia de la Fe lo encontraremos en la comparación que hace el autor entre los seres de la naturaleza y el ser humano afirmando:

No veis hijos el cuidado y la providencia que tiene Dios con los animales, con los pajaritos y con una hormiga? No veis como los animales crian y sustentan a sus hijos, como fabrican sus casitas y las cubren con paja para que sus hijos no se mueran de frío. quién enseñó esto a los pajaritos, quién? Dios Nuestro Señor les dio este conocimiento. quién enseño a estos animales la yerbas buenas para su sustento, quien les dio a conocer las yerbas medicinales para curarse en sus enfermedades? quien? Dios les dió este conocimiento. Pues si Dios les enseño todo esto a los animales que no los crio a su imagen y semejanza como crio al hombre cuya alma es inmortal claro esta que tambien y mucho mejor avia de dar al hombre este conocimiento que le avia de enseñar quien era Dios para que le amase y sirviese. (Avendaño, 1649, f.3) 
A partir de la observación del orden de la naturaleza se busca extraer argumentos para demostrar la existencia de un don que no puede percibirse directamente pero cuya presencia se busca deducir desde lo racional. Aquí se compara el conocimiento que Dios ha puesto en sus criaturas menores para que puedan vivir con el don que está en el alma del ser humano para llevarlo a la salvación. Así, como recurso para aproximarse a un tema de evidente complejidad, se recurre a lo más familiar para la población campesina, el entorno natural, fuente de gran parte de sus creencias.

Considerando que el panteísmo de la población fue uno de los principales desafíos para la evangelización, era fundamental insertar en la prédica la idea de que el perfecto funcionamiento de la naturaleza solo podía explicarse como producto de la creación divina, y que el Dios cristiano era el origen de la armonía perceptible en ella. El discurso, por tanto, se dirige ahora a buscar la comprensión de la naturaleza desde una perspectiva cristiana y convencer a los individuos de que son parte de la creación y de que Dios, a través del don de la fe, habita en ellos.

\subsection{Evidencias de la existencia de la Fe}

Cómo demostrar la existencia de la Fe. Esta aparente contradicción será asumida en el discurso recurriendo a la percepción de los sentimientos, propia de todo ser humano. El amor de Dios a sus criaturas es mostrado a través del sermón cuando se compara con el amor de los padres a sus hijos. Así Dios, al igual que como hacen todos los padres, enseña a sus hijos el camino para que tengan lo mejor en sus vidas y se aparten del mal otorgándoles el don de la Fe.

En primer término, se apela al entendimiento para reconocer que el ser humano cuenta con el don de la Fe y, seguidamente, se afirma la 
importancia que este tiene para dirigir la voluntad y seguir a nuestro guía. A partir de ello, mencionará:

(...) la voluntad con que amamos es potencia ciega ella no conoce a nadie nuestro entendimiento es quien le propone el bien para que ella lo ame y asi con el entendimiento lo conocemos y con la voluntad amamos. Luego si no conociéramos a Dios por la Fe no pudiéramos amarle sobre todas las cosas. (Avendaño, 1649, f. 4)

El asumir la existencia de la $\mathrm{Fe}$ en cada persona lleva al cuestionamiento de qué ocurrió con nuestros antepasados para que desconozcan la Fe. La respuesta aquí trae al discurso a la figura del mal:

(...) ahora os digo que vuestros abuelos por sus pecado grandes fueron perdiendo esta Fe y conocimiento del verdadero Dios y el Demonio como es enemigo de los hombres engaño a nuestros padres Adan y Eva, también engañó a vuestros abuelos y les enseñó que adorasen las huacas y asi perdieron el conocimiento y Fe del verdadero Dios. (Avendaño, 1649, f.4v.)

El discurso busca hacer conscientes de esta realidad a los oyentes insistiendo en el significado de haber recibido el sacramento del bautismo, explicando el por qué de la fórmula bautismal que aquí se recita y, las consecuencias de obrar en contra de ella (Avendaño, 1649).

Una característica esencial de la Fe está en que nos permite creer en lo que no podemos percibir por los sentidos, pues estos pueden equivocarse por ello: 
El mérito de la fe de Dios es creer firmemente los misterios aunque no los veamos y esta fe y creencia la agradece Dios mucho y os la pagara y premiara en el cielo porque seguisteis su fe y guardasteis sus mandamientos. (Avendaño, 1649, f. 5)

La figura de Jesucristo cobra aquí un protagonismo determinante en tanto portador de esta dádiva de Dios al ser humano:

Dios embio a su hijo que es Iessu Christo Señor nuestro al mundo para que nos enseñase esta Fe y nos diese a conocer al verdadero Dios, y los sacerdotes que os enseñamos la fe de Iesu Christo somos unos mensajeros de Dios (Avendaño, 1649, f. 6)

La Fe es mostrada también como la fuente de la fortaleza en el ser I humano, pues solo así se explica la entereza de los mártires frente a terribles tormentos y la vida ejemplar de los santos, ambos testimonio de la existencia de la Fe (Avendaño, 1649).

Observamos que el concepto de Fe que se maneja a lo largo del sermón tiene, entre sus varias acepciones, las siguientes: el de don que nos orienta en el camino hacia el encuentro con Dios, el de fuente de la fortaleza necesaria para enfrentar cualquier adversidad y el de directamente creer en Dios.

Se dirige la atención de los oyentes a las muestras el poder divino, (a través de los milagros de los santos, la presencia de la santa Cruz y las apariciones de la Virgen), frente a las huacas que han sido destruidas, a fin de fortalecer la toma de conciencia de la Fe. 
El argumento final destinado a demostrar la existencia de la Fe se caracteriza por sugerir la cercanía entre la sencillez de los apóstoles y los oyentes, buscando un punto de encuentro entre quienes reciben el mensaje y los elegidos que iniciaron la difusión del cristianismo.

Pues mirad hijos en esto conocereis la omnipotencia de Dios, y la verdad desta Fe y conocimiento suyo pues todos estos milagros los hizo con la predicación de los Apostoles, que eran unos hombres pobres, sin armas ni arcabuzes, sino solamente con la palabra de Dios, que la predicaron en todo el mundo, y mientras mas perseguidos eran los christianos con tormentos y carceles, mas crecian y se multiplicaban. (Avendaño, 1649, f.11)

Como observamos en el sermón se añade también la alusión a la defensa de la $\mathrm{Fe}$, pues se trata de internalizar en los oyentes cómo la persecución, y aun el tormento, habían contribuido a fortalecer y difundir la Fe, muy posiblemente con la intención de brindar argumentos para enfrentar a quienes cuestionaban su conversión al cristianismo.

El sermón finaliza con un llamado a Dios a través de la oración:

Alumbrad señor mi entendimiento con el don de la Fe, para que os conozca por mi Dios y mi Criador, y para que conociendo vuestra Divinidad os ame mi voluntad sobre todas las cosas. O dulce Iesus mio quien nunca os uviera ofendido; O Señor poderoso quien siempre uviera guardado vuestra lei y mandamientos. (Avendaño, 1649, f.11v.) 


\section{El discurso en torno a la existencia de Dios}

\subsection{La naturaleza como referente para la construcción de la idea de Dios}

La concepción de dios en el mundo prehispánico era ciertamente distante de la del Dios cristiano. Sin embargo, como mencionan varios autores, entre ellos el propio Avendaño, la noción de dios creador si estaba presente y será empleada por los predicadores para vincular al pueblo indígena con el cristianismo, tal como lo afirma Marzal "Y esta identificación del Dios creador andino con el cristiano, que sostenían muchos teólogos se predicó efectivamente a los indios al menos en los sermonarios del cuarto periodo de catequización” (Marzal, 1983, p. 198).

La definición del Dios que Avendaño busca proporcionar en este sermón descansa más bien en otorgar a la figura divina una serie de cualidades que la convierten en una presencia activa y determinante para la vida de los cristianos. A decir de Marzal Avendaño, permite “(...) delinear los rasgos que tendrá la imagen de Dios en la espiritualidad andina” (Marzal, 1983, p. 198).

En esta espiritualidad será central la idea de un Dios que va mucho más allá de la creación del mundo, pues en la interpretación que realiza de este sermón afirma que:

No hay duda de que este Dios que se manifiesta en el signo y en el milagro, no es tanto la causa primera de los filósofos cuanto una realidad viva que actúa; por eso Dios jugará un papel importante 
en el control social de la población y en sus motivaciones. (Marzal, 1983, p. 200)

Es en este punto que el tema de lo simbólico adquiere singular importancia, debido a que se trata de verificar la permanente presencia divina. Así, Avendaño expondrá sus argumentos teniendo como base una vez más a la naturaleza, llamando a observar en ella los signos de la divinidad.

La importancia que se atribuye a lo simbólico, sin duda, tiene una estrecha relación con el conocimiento de Avendaño acerca de la concepción de lo divino en el mundo precristiano, siempre ligado a hechos concretos.

Pero no solo se tratará de buscar señales sino que la naturaleza en su conjunto habrá de ser vista como la mayor evidencia de la existencia de Dios.

Pues siendo esto asi, hombre abre tus ojos y mira este mundo, que es como un gran palacio y casa de Dios muy hermosa. Mira los techos desta casa que son esos cielos, mira esta tierra tan grande, mira este mar tan profundo, mira que esta casa no solamente esta hermoseada con alabastros y piedras preciosas, diamantes, rubies, oro, plata y otras piedras preciosas, sino con este sol, hermoso y resplandeciente, y con esta luna tan hermosa, con el Luzero y estrellas brillantes. (Avendaño, 1649, f. 15)

Aquí no solo se alude a la belleza de la creación sino a la armonía que reina en ella, donde todo parece estar dispuesto para cuidar y proveer todas las necesidades del ser humano. 
mira como Dios en esta casa puso todas estas sus criaturas, para que nos sirviesen, unas dellas nos alumbran como el Sol, la Luna y la Estrellas, otras nos dan de comer, como son los pajaros, animales y pezes y los arboles y las plantas, otras nos alegran y entretienen como son las flores, otras nos visten y cubren como los vestidos y las casas y otras son para nuestro descanso y alivio como la sombra para el sol, los manantiales de agua para la sed, los medicamentos 'para las enfermedades, y para aliviar el cansancio del camino, nos dio los caballos en que caminamos... (Avendaño, 1649, f. 15)

La reflexión se lleva ahora a estimular el cuestionamiento de los oyentes frente a la realidad que perciben.

(...) viendo esto que os he dicho y aun no aviendo entrado a lo interior desta casa de Dios y aun no aviendo visto claramente al mismo Dios que vive en lo alto del cielo, que es el Señor deste palacio, no dirás en tu corazon: no es posible que esta casa sea hecha de si misma, no es posible que todos estos criados nos sirvan de si mismos, sin orden ni disposicion de alguien. Algun gran señor fabrico aquesta casa, algun Gran Señor mando que estas sus criaturas sirvan al hombre, en diferentes tiempos. Pues este Gran Señor, hijos es el que la Fe nos enseña que es Dios. (Avendaño, 1649, f.15V) 


\subsection{El orden natural como expresión de la presencia de Dios}

La existencia de Dios no solo buscará demostrarse a partir de la belleza y de la acogida que brinda la naturaleza al ser humano, la armonía que reina en ella será también uno de los elementos esenciales para fundamentar la existencia de un creador. El autor describe, como veremos, una jerarquía entre los seres a partir del criterio de la posesión de vida. Este argumento colocaba a las antiguas deidades en una posición muy por debajo del ser humano, al interior de una creación donde cada quien ocupa el lugar que le corresponde y todo funciona en perfecta armonía.

Veamos pues mas. no veis como todas las cosas conservan y tienen cada una un lugar señalado que cada una sin revolverse con la otra se esta en su puesto y lugar? Unas tienen nomas que ser, sin tener vida, como los cielos, la tierra, la mar, el aire, el sol, la luna, las estrellas y las piedras. Otras tienen el primer grado de vida, que viven y no sienten como son los arboles y las plantas y las flores, que su vida es crecer y aunque las [cultiven] no tienen sentido. Otras tiene vida y cinco sentidos porque sienten, ven, oyen, huelen, tocan y gustan como son los animales, los pajaros, y los peces, pero no hablan como los hombres, ni discurren, ni su alma es inmortal, y los hombres teniendo ser viven, y viviendo sienten y sintiiendo discurren y hablan y su alma es inmortal. (Avendaño, 1649, f.16 v.)

Y aquí nuevamente el llamado a la reflexión: 
Veamos quien puso todas las criaturas cada una en sus lugares. Dime: el sol de si mismo alumbra siempre? Y los cielos por su misma virtud permanecen sin que se destruyan? Quien hizo que unas cosas tengan ser, otras vivan, otras sientan y otras entiendan? (Avendaño, f.17)

Vemos, así, cómo en la prédica se apela a la razón dirigida a la observación de la realidad de lo cotidiano, de aquello que estaba presente en el día a día, con el fin de repensarlo como un todo armónico donde el ser humano ocupaba el lugar preferente.

\subsection{Argumentos para la concepción de un Dios espiritual}

Todas las argumentaciones anteriores están dirigidas a mostrar a Dios como el creador y ordenador del mundo en el que los seres humanos, hechos a su imagen y semejanza, pueden vivir como soberanos de todo lo creado.

Pero cómo explicar el carácter espiritual de Dios. Para ello, se recurrirá nuevamente a la naturaleza y a los fenómenos que ocurren en ella pero que no es posible ver.

Dezidme no crreys firmemente que los arboles y las plantas crecen? Esto no podeis negarlo. Es verdad Padre. Pues dezidmen aora. has visto tu con tus ojos de la manera que crece el arbol? no lo puedes aver visto porque aunque estes junto al arbol de dia y de noche, no podras ver con tus ojos como crece. Verdad es que veras que tu mayz esta agora mas crecido, que estava el mes pasado? aunque lo esteis mirando no podras ver con tus ojos del 
modo y manera que el crece y se levanta de la tierra. (Avendaño, 1649, f. 18)

De la percepción de lo invisible en el exterior, el sermón gira al interior del ser humano evidenciando aquellos aspectos que aunque carezcan de corporeidad es imposible negar que existen, diciendo:

Oyeme otra razon. No sabes tu de cierto que tienes entendimiento que estas pensando en Cuzco y en Castilla y con el te andas paseando por todo el mundo? No sabes tambien que tienes voluntad con que amas y codicias los bienes agenos? No sabes que dentro de tu cuerpo tienes alma con que vives andas y comes? Pues dime agora as visto a tu entendimiento de que color es? As visto a tu voluntad de que forma es? As visto a tu alma? Pues que? Seria bien dezir que, porque no as visto a tu entendimiento, ni a tu voluntad ni a tu alma que por eso no tenias entendimiento, ni alma, ni voluntad? No seria esto cosa de burla. (Avendaño, 1649, f. 18)

Entendimiento, voluntad y alma; aspectos que en el mensaje cobran gran importancia por tratarse de cualidades superiores del ser, siendo justamente su condición de imperceptibles la que hace que estén más próximas al Creador "porque Dios en quanto Dios no tiene cuerpo ni carne todo es espiritu Dios Vuestro Señor espiritu puro en quanto Dios sin mescla de cuerpo, porque Dios en su ser y perfeccion excede infinitamente al alma” (Avendaño, 1649, f.18 v.). 


\section{Conclusiones}

$\mathrm{P}$ odemos concluir, a partir de la reflexión en torno a la explicación que realiza el autor acerca de los temas de la Fe y la naturaleza de Dios que, en primer lugar, el mensaje se dirige al entendimiento, a la capacidad de comprensión de los oyentes. Destaca, de esta forma, la necesidad de la comprensión para guiar la voluntad hacia el bien.

Seguidamente, concluimos que existe una relación de interdependencia entre el concepto de Dios que se busca transmitir y la naturaleza. No es posible explicar a Dios sin resaltar la belleza y armonía de la creación y viceversa. Siendo el ser humano el punto culminante de la obra divina.

El discurso pasa de lo concreto y observable hacia los aspectos de la realidad que no podemos observar pero que indudablemente existen para luego conducir al oyente hacia nociones más abstractas como la realidad de lo invisible en el interior del ser humano.

Finalmente, la ampliación de la noción de realidad hacia el ámbito de lo invisible. Partiendo de hechos que no se pueden percibir directamente a través de los sentidos hacia nociones más abstractas, permite la formulación de una concepción omnipresente y actuante de Dios que guía al ser humano desde su interior. 


\section{REFERENCIAS}

Acosta, A. (2014). Las prácticas coloniales de la Iglesia en el Perú. Aconcagua - Instituto de Estudios sobre América Latina (IEAL) Sevilla.

Acosta, J. [1589] (1954). Historia naturaly moral de Indias. Biblioteca Virtual Miguel de Cervantes. Recuperado de http://www.cervantesvirtual. com/obra-visor/historia-natural-y-moral-de-las-indias--0/html/

Avendaño, F. (1649). Sermones de los misterios de nuestra Santa fe católica en lengua castellana y la general del inca. Impugnase los errores particulares que los indios han tenido. Lima, Perú: Imp. Jorge López de Herrera.

Arriaga, J. (1621). La extirpación de la idolatría en el Perú. Biblioteca Virtual Miguel de Cervantes. Recuperado de http://www.cervantesvirtual. com/obra-visor/la-extirpacion-de-la-idolatria-en-el-peru--0/html/ ff49f4c0-82b1-11df-acc7-002185ce6064_16.html

Cerdán, F. (1996). La oratoria sagrada en el siglo XVII. Un espejo de la sociedad. En Actas de los Congresos de la Asociación Internacional Siglo de Oro (AISO). Centro Virtual Cervantes. Recuperado de https:// seminariofranciscanos.files.wordpress.com/2013/10/la-oratoriasagrada-del-siglo-xii-francis-cerdc3a1n.pdf

Duviols, P. (2003). Procesos y visitas de idolatrías. Cajatambo siglo XVII. Lima, Perú: Pontificia Universidad Católica del Perú, Instituto Francés de Estudios Andinos.

Guibovich, P. (1993). La carrera de un visitador de idolatrías en el siglo XVII: Fernando de Avendaño 1580?-1655. En Catolicismo y extirpación de idolatrías siglos XVI - XVII pp. 169 - 240. Cusco, Perú: Centro 
de Estudios Regionales Andinos Bartolomé de Las Casas Ramos y Urbano.

Lisi, F. (2008). El Tercer Concilio de Limay su significación en la aculturación de las poblaciones indígenas de la América del Sur. Guaraguao, 12(28) (Summer, 2008), 71-74. Recuperado de https://www.jstor. org/stable/25596636?readnow=1 \&seq=1\#page_scan_tab_contents

Marzal, M. (1983). La transformación religiosa peruana. Lima, Perú: Fondo Editorial de la Pontificia Universidad Católica del Perú.

Pease, F. (1991). La iglesia, la evangelización. En Perú hombre e historia (T. II) (pp. 331-347). Lima, Perú: Edubanco. 